nehmen. 1990 gründete sie gemeinsam mit ihrem Ehemann Dr. Alexander Vieler die Dr. Vieler \& Partner GbR, eine überregional tätige Sozietät für Wirtschaftsprüfung mit Hauptsitz in Chemnitz. Die Sozietät versteht sich als Partner für die Prüfung und Steuerberatung ihrer überwiegend mittelständischen Mandanten. Ein besonderer Schwerpunkt liegt in der Kommunalwirtschaft. Hier unterstützt die Sozietät Gemeinden und Hochschulen bei der Umsetzung des neuen kommunalen Finanzmanagements.

Regina Vieler ist dem djb vor Kurzem beigetreten..

\section{Gisela Wild}

zum 80. Geburtstag. Gisela Wild wurde am 18. September 1932 in Bad Warmbrunn (Riesengebirge) geboren. Nach der Vertreibung aus Schlesien nach Kriegsende zog die Familie nach Konstanz. 1952 begann Gisela Wild ihr Jurastudium in Freiburg. Ihr Referendariat absolvierte sie in Baden-Württemberg, Köln und Hamburg. 1960 promovierte sie.1961 wurde sie als 16. Rechtsanwältin in Hamburg zugelassen und fing in der Kanzlei Prof. Bussmann, Dr. Droste an. Sie spezialisierte sich auf gewerblichen Rechtsschutz, Urheber- und Presserecht.

1964/65 lebte Gisela Wild in Paris, wo ihr Sohn geboren wurde. 1970 trat sie als erste Partnerin in die Kanzlei von Berenberg-Gossler, Frhr. von Gleichenstein in Hamburg ein, die 1990 auf ihre Initiative mit der Kanzlei Wessing in Düsseldorf und Zimmermann in München fusionierte, 2002 erfolgte der internationale Zusammenschluss zur Kanzlei Taylor Wessing.

2005 wurde sie von der Bürgerschaft als Hamburgische Verfassungsrichterin gewählt. Gisela Wild wurde einer breiten Öffentlichkeit durch das sogenannte Volkszählungsurteil und den Emma-Prozess bekannt. 1996 erhielt sie das Bundesverdienstkreuz, 2010 den Maria-Otto-Preis des DAV.

Gisela Wild ist seit 1976 Mitglied des djb, 1977 bis1979 war sie 2. Vorsitzende, bis 1983 Vorstandsmitglied. Ehrenamtlich engagiert sie sich für SterniPark e.V.

\section{Geburtstage}

(Juli bis September 2012)

\section{Jahre}

Dr. Ingelore Seidel Rechtsanwältin Bonn

- Dr. Gisela Wild Rechtsanwältin Hamburg

1977 bis 1979 2. Vorsitzende des djb 1979 bis 1983 Mitglied des erweiterten Bundesvorstands

\section{Verstorben}

\section{Jahre}

- Dr. Annemarie Volkheimer-Röhrig Rechtsanwältin i.R.

Memmingen

\title{
Ehrennadeln: ein Teil der Anerkennungskultur im djb
}

\section{Anke Gimbal \\ Geschäftsführerin des djb, Berlin}

Unter dem Stichwort „Wertschätzung von Mitgliedern“ wurde im Laufe der Jahre in vielen Bundesvorstandssitzungen darüber diskutiert, wie man das am besten umsetzt. In seiner Sitzung am 22./23. November 2003 beschloss der Bundesvorstand schließlich die Einsetzung einer Nichtständigen Kommission mit dem Auftrag, zu folgenden Punkten Konzepte zu entwickeln und dem Vorstand geeignete Vorschläge vorzulegen: 1. Auslobung eines djb-Stipendiums, 2. Vergabe eines djb-Preises für eine besonders ausgezeichnete Juristin, 3. Entwurf einer Ehrenordnung des djb. Die Kommission wurde sodann für ein Jahr eingesetzt. Die Mitglieder, Rechtsanwältin und Senatorin für Justiz a.D. Dr. Lore Maria Peschel-Gutzeit (Vorsitzende), Präsidentin des Bundespatentgerichts i.R. Antje Sedemund-Treiber, Rechtsanwältin und Bürgermeisterin a.D. Gabriele Klug, Justitiarin Ulrike Gauderer und Rechtsanwältin Sonja Riedemann, LL.M. (LSE), legten Ende 2004 einen umfangreichen Abschlussbericht vor. Von den Empfehlungen 


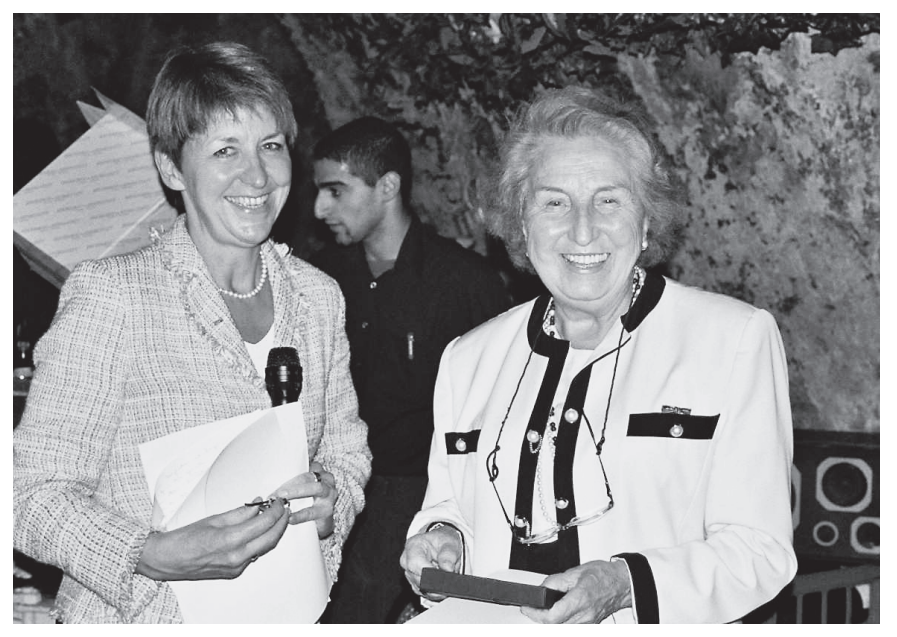

wurden viele umgesetzt - so zum Beispiel die Einrichtung des Amtes der Ehrenpräsidentin und die Auslobung eines djbPreises - und eben auch die Vergabe von Ehrennadeln. Die Kommission hatte die Vergabe von djb-Ehrennadeln für langjährige Mitglieder in drei Stufen vorgeschlagen in Bronze nach über 15-jähriger Mitgliedschaft, in Silber nach über 25-jähriger Mitgliedschaft, in Gold nach über 35-jähriger Mitgliedschaft. Der Bundesvorstand entschloss sich, djb-Ehrenzeichen erstmals anlässlich des 36. Kongresses in Trier am 24. September $2005 \mathrm{zu}$ verleihen. Zunächst wurden ganz unterschiedliche Ideen, wie diese Nadeln denn aussehen könnten, diskutiert und Entwürfe in Auftrag gegeben. Auch die Mitglieder wurden gebeten, Vorschläge für ein djb-Ehrenzeichen einzureichen. Das Design einer Berliner Goldschmiedin fand die größte Zustimmung, denn auch die Kommission hatte dem Bundesvorstand ans Herz gelegt, hochwertiges Material zu wählen. Hergestellt wurden schließlich goldene djb-Anstecknadeln für alle Mitglieder und eine Variante mit einer Verzierung in Weißgold für aktive Mitglieder, die von den Mitgliedern erworben werden können. Außerdem wurden zwei Ausführungen goldener Anstecknadeln mit einem weißen Brillanten (für 35 Jahre Mitgliedschaft) und einem weiteren blauen Diamanten (für 50 Jahre Mitgliedschaft) erworben, die ausschließlich verliehen werden. Der Bundesvorstand wich insoweit von den Vorschlägen der Kommission ab.

Etwa 65 Frauen mit entsprechend langer Mitgliedschaft waren Anfang 2005 in der djb-Mitgliederdatei gelistet und wurden zur Mitgliederversammlung 2005 in Trier eingeladen. Da diese Mitgliederdatei erst Mitte der Neunzigerjahre entstanden war und die Übertragung der Daten in EDV aus den zuvor verwendeten Karteikästen fehleranfällig war (z.B. konnten Unterbrechungen der Mitgliedschaft damals nicht erfasst werden), gab es außerdem Aufrufe in den „aktuellen informationen“ an langjährige Mitglieder, sich bei der Geschäftsstelle zu melden und ihr Eintrittsjahr in den djb mitzuteilen. Daten vor 1960 sind in der djb-Mitgliedsdatei leider gar nicht enthalten.

Die meisten angesprochenen Kolleginnen wählten anstelle der Reise nach Trier das alternative Angebot, nämlich die Übergabe der Nadeln im Rahmen kleinerer oder auch $\varangle$ Margret Diwell (zum Zeitpunkt der Verleihung der Ehrennadeln im Rahmen des Gemeinsamen Abends nach der Mitgliederversammlung gerade Past President des djb geworden) überreicht Dr. Helga Stödter (Legationsrätin, geb. 1922, verst. 2011) die Nadel für mehr als 50 Jahre Mitgliedschaft im djb. (Foto: Katja Schwind)

größerer Feiern der Regionalgruppen bzw. Landesverbände oder auch Besuche durch die Vorstandsmitglieder zu Hause. Doch immerhin wurde in Trier den ersten fünf Kolleginnen die Nadel für 35 Jahre Mitgliedschaft und drei weiteren Kolleginnen eine solche für 50 Jahre Mitgliedschaft verliehen. Dies waren: Antje Daniels-Wiesmann, Dr. Anne van Hees, Dr. Heidi Lambert-Lang, Ingrid Baer, Dr. Helga Pense (35 Jahre) und Dr. Helga Stödter, Dr. Hertha Engelbrecht sowie Dr. Barbara Höynck-Lüthgen (50 Jahre). Ein Bericht von Margret Diwell, djb-Präsidentin von 2001 bis 2005, ist in den „aktuellen informationen “ $1 / 2006$, Seite 56 f. dokumentiert.

Seitdem haben sich die Ehrennadeln im Verbandsleben etabliert und bewährt. Zu Anfang eines jeden Jahres erhalten die Vorstände der Regionalgruppen und der Landesverbände (jene ohne Regionalgruppen) von der Geschäftsstelle eine Liste derjenigen Mitglieder, die das entsprechende Mitgliedsalter erreicht haben. Die Landesverbände und Regionalgruppen entscheiden, wie sie weiter verfahren möchten - und zwar auch unter Einbeziehung der Wünsche der zu Ehrenden. Manche Mitglieder freuen sich sehr über ihre Nadel, andere möchten einfach keine. Einige wählen eine größere oder kleinere Feier, andere möchten ihre Nadel einfach mit der Post zugeschickt bekommen. Wer die „aktuellen informationen“ bzw. die djbZ aufmerksam liest, findet immer einmal wieder einen Bericht oder ein Bild anlässlich der Verleihung einer Ehrennadel an ein langjähriges Mitglied. Insgesamt wurden bis heute $41 \mathrm{Na}-$ deln für 35 Jahre und 26 Nadeln für 50 Jahre djb-Mitgliedschaft verliehen.

Langjährige Mitglieder haben zwangsläufig eine reiche Verbandserfahrung, meist ein langes Berufsleben. Einige haben beeindruckende Karrieren aufzuweisen und können den jüngeren Mitgliedern als Vorbild dienen. Niemand muss das Rad neu erfinden. Denn viele Probleme - karrieretechnisch oder bei der Organisation von Beruf und Privatleben - wurden schon einmal von anderen gelöst. Es lohnt sich daher zuzuhören und hinzuschauen, sobald sich die Gelegenheit etwa bei der Ehrung langjähriger Mitglieder ergibt. Etwas erschreckend ist es jedoch, wenn man dann gelegentlich feststellt, dass sich in manchen Bereichen in den vergangenen 50 Jahren einfach nichts geändert hat. Der djb mit seinem Ziel der Gleichberechtigung und Gleichstellung der Frau in allen gesellschaftlichen Bereichen wird wohl so bald nicht überflüssig werden. Eines kommt jedoch heutzutage sicher nicht mehr vor: bei Jura-Vorlesungen als einzige Frau im Saal zu sitzen. 